\title{
A NEW FORMULATION OF THE GENERALIZED TODA LATTICE EQUATIONS AND THEIR FIXED POINT ANALYSIS VIA THE MOMENTUM MAP
}

\author{
ANTHONY M. BLOCH, ROGER W. BROCKETT, AND TUDOR S. RATIU
}

\section{INTRODUCTION}

The Toda flows are examples of integrable Hamiltonian systems which are of great interest from the point of view of pure mathematics as well as in applications. They originated as a description of a 1-dimensional lattice of particles with exponential interaction [29]. Flaschka [14] found a Lax pair for the equations and Moser [25] analyzed the dynamics and scattering behavior of the system. Adler [1], Kostant [21,22] and Symes [26,27] generalized the results to arbitrary Lie algebras. Symes [28] also showed that the Toda equations have a surprising connection with numerical analysis: the time-1 map of the Toda flow is equivalent to the QR algorithm for diagonalizing symmetric matrices (see also $[11,13,17,23,28])$.

Our point of view in this paper also originated from analyzing a problem of numerical importance. In $[8,9]$ Brockett studied a critical point problem arising from geometrical matching problems in computer vision. He derived the gradient flow associated with the critical point problem and showed that the equations had an elegant Lax pair form. In the case of the orthogonal group these equations give a new method of solving the symmetric eigenvalue problem. In [4] Bloch observed that a particular case of these equations gives the Toda equations in the Flaschka form.

Received by the editors April 12, 1989 and, in revised form, December 4, 1989.

1980 Mathematics Subject Classification (1985 Revision). Primary 34A05; Secondary 22E46.

The first author is supported in part by NSF Grant DMS-87-01576 and AFOSR Grant AFOSR-ISSA-87-0077 and the U.S. Army Research Office through the Mathematical Sciences Institute of Cornell University.

The second author is supported in part by AFOSR grant AFOSR-96-0197, by U.S. Army Research Office grant DAAL03-86-K-0171, and by NSF grant CDR-8500108 .

The third author is supported in part by NSF Grant DMS-870318-01. 
In this paper we announce essentially two results. Firstly, we show how Brockett's equations $\dot{L}(t)=[L(t),[L(t), N]]$, which can be defined for any real or complex algebra, specialize for suitable $N$ and suitable initial conditions to the generalized Toda lattice equations (in the sense of Kostant and Symes) on any semisimple Lie algebra. In fact, the tridiagonal matrices of the Toda lattice occur naturally as one of a set of invariant subspaces for these equations. Moreover, using the compact real form of the algebra, we show how this approach provides a direct formulation of the Toda lattice equations as a gradient flow. This formulation is quite different from that given by Moser in [25].

Secondly, again using the compact real form, we show how the fixed points of the equations are the vertices of a convex polytope which is the image of a momentum map associated with the problem. Further, we show that the projection of the Toda flow defined by the momentum map lies in the interior of the polytope. This provides some insight into the convexity results that were observed to be associated with the Toda lattice problem by Deift, Nanda, and Tomei [13] (see also van Moerbeke [31] and Tomei [30]).

\section{The generalized Toda lattice equations AND GRADIENT FLOWS ON LIE GROUPS}

In this section we show how the generalized Toda lattice equations (see $[22,29])$ may be written as an equation of the form $\dot{L}(t)=[L(t),[L(t), N]], N$ being fixed. Remarkably, this shows that these flows not only are Hamiltonian but also are gradient flows on a submanifold of an adjoint orbit of a compact Lie group.

Consider first the general case where $L$ and $N$ are taken to be elements of any Lie algebra $\mathscr{G}$ defined over the real or complex field. The differential equation $\dot{L}=[L,[L, N]]$ then evolves in $\mathscr{G}$. This equation, introduced in [9], has been investigated in the special case where the Lie algebra is the unitary algebra and $L$ and $N$ are symmetric (and thus are elements of $u(n)$ after multiplication by i). In that case the equation evolves in the subspace of symmetric matrices.

However, there are other invariant subspaces for the nonlinear operator $[L,[L, N]]$ and in the proposition below we describe an invariant subspace in a Lie algebra which enables us to formulate the generalized Toda lattice equations in the form $\dot{L}=[L,[L, N]]$. 
Proposition. (i) Let $r \in \mathbb{R}$ and let $\mathscr{G}$ be a Lie algebra. Let $N \in \mathscr{G}$ and suppose $(\mathrm{ad} N)^{2}$ has an eigenvalue $r^{2}$ with corresponding eigenspace $V$ in $\mathscr{G}$. Let $\mathscr{G}_{N}$ be the centralizer of $N$. Then $\mathscr{G}_{N} \oplus V$ is an invariant subspace for the nonlinear operator on $\mathscr{G}$ given by $L \rightarrow[L,[L, N]], L \in \mathscr{G}$.

(ii) Suppose $e$ is an involutive Lie algebra automorphism of $\mathscr{G}$. Let $\mathscr{G}_{\text {even }}$ and $\mathscr{G}_{\text {odd }}$ be the +1 and -1 eigenspaces of $e$. Then if ad $N$ maps $\mathscr{G}_{\text {odd }}$ into $\mathscr{G}_{\text {even }}$, it follows that $\left(\mathscr{G}_{N} \oplus V\right) \cap \mathscr{G}_{\text {odd }}$ is an invariant subspace for the operator $[L,[L, N]]$.

The proposition can be proved by straightforward computation using the Jacobi identity. The main point here is that the Toda flows evolve in a subspace of the form defined in part (ii) of the proposition with $r^{2}=1$. (For the standard representation of $s l(n)$ this is just the subspace of symmetric tridiagonal matrices.) This subspace is described explicitly in the theorem below.

Now, in what follows, let $\mathscr{G}_{u}$ be a compact Lie algebra, $\mathscr{A}$ a maximal abelian subalgebra, and $\mathscr{G}$ the complexification of $\mathscr{G}_{u}$, and choose $\mathscr{H}=\mathscr{A} \oplus i \mathscr{A}$ as the Cartan subalgebra of $\mathscr{G}$. We let $\Delta$ denote the system of roots of $\mathscr{G}$ defined by $\mathscr{H}$ and choose $\left\{h_{j}, e_{\alpha} \mid j=1, \ldots, \ell, \alpha \in \Delta\right\}$, a Chevalley basis of $\mathscr{G}$, with $h_{j} \in \mathscr{A} ; \alpha_{1}, \ldots, \alpha_{\ell}$ denote the simple roots. (See, for example, [24].)

Let $G$ and $G_{u}$ denote the Lie groups with Lie algebras $\mathscr{G}$ and $\mathscr{G}_{u}$, respectively. We then have the following:

Theorem 1. (i) Let $N \in \mathscr{G}_{u}$. The equation on $\mathscr{G}_{u}$

$$
\dot{L}=[L,[L, N]]
$$

is the gradient vector field of $\kappa(L, N), \kappa$ being the Killing form, on the adjoint orbit $\mathscr{O}$ of $\mathscr{G}_{u}$ containing the initial condition $L(0)=$ $L_{0}$. Its flow is the image under the quotient map $G_{u} \rightarrow \mathscr{O}=G_{u} / H$, $H$ being the stabilizer of $L_{0}$, of a gradient flow on the group $G_{u}$. (ii) If $N$ is $i$ times the sum of the simple coweights of $\mathscr{G}$, then for

$$
L=\sum_{j=1}^{\ell} i b_{j} h_{j}+\sum_{j=1}^{\ell} i a_{j}\left(e_{\alpha_{j}}+e_{-\alpha_{j}}\right),
$$

equation (1.1) gives the generalized Toda lattice equations on the level set of all the integrals of the Toda flow. Explicitly, $N$ is given by

$$
N=\sum_{j} i x_{j} h_{j}
$$


where $\left(x_{1}, x_{2}, \ldots, x_{\ell}\right)$ is the unique solution of the system

$$
\sum_{j} x_{j} \alpha_{i}\left(h_{j}\right)=-1, \quad i=1, \ldots, \ell,
$$

$\alpha_{i}\left(h_{j}\right)$ being the Cartan matrix of $\mathscr{G}$.

Sketch of Proof. (i) Define the smooth function $\Theta \rightarrow \kappa\left(Q, \operatorname{Ad}_{\Theta} N\right)$ on $G_{u}$ for fixed $Q$ and $N$. The gradient flow of this function relative to the left-invariant Riemannian metric on $G_{u}$, whose value at the identity is minus the Killing form, has the expression (see [8] for the unitary case)

$$
\dot{\Theta}=\Theta \cdot\left[\operatorname{Ad}_{\Theta^{-1}} Q, N\right]
$$

where $\Theta \cdot P$ denotes the left translation of $P$ by $\Theta$.

Projecting onto the adjoint orbit containing $Q$ by setting $L=$ $\operatorname{Ad}_{\Theta^{-1}} Q$, we find

$$
\dot{L}=[L,[L, N]] \text {. }
$$

Further, the function $\kappa(L, N)$ defined on this adjoint orbit endowed with the "standard" or "normal" metric (see Atiyah [2]) also has the gradient flow (1.7). Explicitly this metric is given in the following way. Decompose orthogonally, relative to $-\kappa()=$, $\langle\rangle,, \mathscr{G}=\mathscr{G}^{L} \oplus \mathscr{G}_{L}$, where $\mathscr{G}_{L}$ is the centralizer of $L$ and $\mathscr{G}^{L}=\operatorname{Im}$ ad $L$. For $X \in \mathscr{G}_{u}$ denote by $X^{L} \in \mathscr{G}^{L}$ the orthogonal projection of $X$ on $\mathscr{G}^{L}$. Then set the inner product of the tangent vectors $[L, X]$ and $[L, Y]$ to be equal to $\left\langle X^{L}, Y^{L}\right\rangle$.

(ii) From (1.2) and (1.3)

$$
[L, N]=\sum_{i=1}^{\ell} \sum_{j=1}^{\ell} x_{j} a_{i} \alpha_{i}\left(h_{j}\right)\left(e_{\alpha_{i}}-e_{-\alpha_{i}}\right) .
$$

But for the generalized Toda flow [27], $\dot{L}=[B, L]$, where

$$
B=\sum_{i=1}^{\ell} a_{i}\left(e_{\alpha_{i}}-e_{-\alpha_{i}}\right) .
$$

Hence $[L, N]=-B$ if

$$
\sum_{j=1}^{\ell} x_{j} \alpha_{i}\left(h_{j}\right)=-1 .
$$

It is a standard result that $\alpha_{i}\left(h_{j}\right)$ is the Cartan matrix of $\mathscr{G}$. 
Now let $\check{\alpha}_{i}=2 \alpha_{i} /\left(\alpha_{i}, \alpha_{i}\right)$, where (, ) denotes inner product, be a dual root and let $\check{\lambda}_{i}$, where $\left(\check{\lambda}_{i}, \alpha_{j}\right)=\delta_{i j}$, be the corresponding dual weight. Set $h_{\check{\delta}}=\sum_{i=1}^{\ell} \check{\lambda}_{i}$. Then $\left[h_{\check{\delta}}, e_{\alpha_{i}}\right]=1$ and the $x_{i}$ are the coefficients of $-h_{\check{\delta}}$ in the original root basis. Note that $N=-i h_{\check{\delta}}$ is also the Cartan part of a principal $s l(2)$ (see [19]).

The coefficients $x_{i}$ (i.e., the solutions of equation (1.4)) for all the semisimple Lie algebras may be found by consulting [7].

We remark that the Toda lattice is actually gradient on its restriction to the isospectral set, since it is the restriction of a gradient vector field on a $G_{u}$ orbit. There is no suitable metric off the isospectral set. This is in agreement with Moser's form of the gradient flow (see [25]) given by

$$
\frac{d r_{k}}{d t}=-\frac{\partial V}{\partial r_{k}}, \quad k=1, \ldots, n
$$

where

$$
V=\frac{\sum_{k=1}^{n} \lambda_{k} r_{k}^{2}}{2 \sum_{k=1}^{n} r_{k}^{2}} .
$$

Here the $\lambda_{i}$ are constant and satisfy $\lambda_{1}<\lambda_{2}<\cdots<\lambda_{n}$, and the $r_{k}$ are strictly positive and satisfy $\sum_{k=1}^{n} r_{k}^{2}=1$.

We remark also that for $s l(\ell+1)$ our function $\kappa(L, N)$ is (modulo a trivial normalization) the Morse function discussed in $[12,15,30]$ and used to analyze the topology of isospectral sets of Jacobi (symmetric, tridiagonal) matrices. We have shown that this Morse function actually gives the Toda flow as its gradient flow with respect to a suitable metric and, moreover, we have given a natural generalization of this function to arbitrary semisimple Lie algebras.

\section{The TODA FLOWS AND THE MOMENTUM MAP}

In [13] (see also [30, 31]) it was observed that the Toda flow could be viewed in a topological sense as lying inside a convex polytope. As discussed in the introduction, here we give some insight into this observation for the generalized Toda lattice equations in terms of the theory of the convexity of the momentum map as developed by Schur-Horn [18], Kostant [20], Atiyah [2], and Guillemin and Sternberg [16]. The key that enables us to do this is the observation in $\S 1$ that the flow of the Toda lattice may 
be viewed as a gradient flow on a submanifold of an adjoint orbit of the compact real form of the Lie algebra.

As observed by Brockett in [9], one can in fact view the equation $\dot{L}=[L,[L, N]]$ in the Hermitian case as solving a linear programming problem on the convex set defined by the Schur-Horn theorem. This also makes contact with the problem of minimizing the Total Least Squares function (see [3, 4, 10]).

Theorem 2. (i) Let $\mathscr{O}$ be a (co) adjoint orbit of $G_{u}$ in $\mathscr{G}_{u}$ and consider the gradient vector field $\dot{L}=[L,[L, N]], L(0) \in \mathscr{O}$, with flow $F_{t}$ for $N$ a fixed regular element in $\mathscr{G}_{u}$. The set of equilibria of this vector field coincides with $\mathscr{O} \cap \mathscr{A}$ where $\mathscr{A}$ is the Cartan subalgebra of $\mathscr{G}_{u}$ containing $N$. This set $\mathscr{O} \cap \mathscr{A}$ consists of a single Weyl group orbit. The convex hull of these equilibria is a compact polytope $\mathscr{P}$, which is the image of $\mathscr{O}$ under the momentum map $\pi: \mathscr{O} \rightarrow \mathscr{A}$ defined by the adjoint $T$-action on $\mathscr{O}$, where $T$ is the maximal torus in $G_{u}$ obtained by exponentiating $\mathscr{A}$. The momentum map $\pi$ is the orthogonal projection of $\mathscr{O}$ onto $\mathscr{A}$ and $\pi\left(F_{t}(\mathscr{O})\right)$ lies entirely in $\mathscr{P}$.

(ii) If $N$ and $L$ are chosen as in Theorem 1 (ii), $\dot{L}=[L,[L, N]]$ becomes the Toda lattice equations defined on the level set of all constants of the motion and the projection of the Toda flow on $\mathscr{A}$ lies in the interior of the polytope given in (i).

Sketch of Proof. $T$ acts on $\mathscr{O}$ by the (co)adjoint action, defining the momentum map $\pi: \mathscr{O} \rightarrow \mathscr{A}=\mathbb{R}^{\ell}$ which is the orthogonal projection of $\mathscr{O}$ onto $\mathscr{A}$ (relative to the metric $-\kappa($,$) on \mathscr{G}_{u}$ ). The image of $\pi$ is a convex compact polytope, which is the convex hull of the critical values of $\pi$. These critical values coincide with the Weyl group orbit $\mathscr{O} \cap \mathscr{A}$ (see $[2,16,18,20]$ ).

To prove the theorem we will show that the equilibria of the vector field $\dot{L}=[L,[L, N]]$ on $\mathscr{G}_{u}$ necessarily lie in $\mathscr{A}$. (See [9] for the unitary case.) Note firstly that the solutions of the equation $\dot{L}=[L,[L, N]]$ exist for all time since the flow is the projection of a flow from a compact group. We will now show that $\lim _{t \rightarrow \infty} L(t)$ must be in $\mathscr{A}$. Observe that

$$
\frac{d}{d t}(\kappa(L, N))=\kappa(N,[L,[L, N]])=-\kappa([L, N],[L, N]) .
$$

Hence $\kappa(L, N)$ is monotonic increasing along the flow and is bounded above, since $L(t)$ lies in the coadjoint orbit of a compact group. Thus $\kappa(L, N)$ has a limit and its derivative goes to 
zero. But we can see that its derivative vanishes only if $L$ and $N$ commute and, since $N$ is regular, $L$ must lie in $\mathscr{A}$.

We remark that the Toda lattice equations on the level set of all constants of the motion have no equilibria, but if one considers these equations defined on the closure of the Toda phase space, then the equilibria are given by the Weyl group orbit as stated in Theorem 2. From Theorem 2 we can determine that the number of equilibria of the vector field $\dot{L}=[L,[L, N]]$ in $\mathscr{O}$ equals the order of the Weyl group.

Detailed proofs and further analysis of the work announced here will appear in [6]. We emphasize again that the image of the Toda flow under the momentum map lies in the interior of the polytope defined in Theorem 2; it does not define a flow everywhere in the polytope. We also point out that with Flaschka in [5] we have analyzed the Kähler geometry of the Toda flows yielding another convexity theorem. In this situation the Toda flow does map via the momentum map to a flow on the polytope.

\section{ACKNOWLEDGMENTS}

We gratefully acknowledge all our discussions with $\mathrm{H}$. Flaschka and his invaluable input in the final form of this work. We also thank J. Marsden for several useful conversations. Finally, we would like to thank the anonymous referees for their very helpful suggestions.

\section{REFERENCES}

1. M. Adler, On a trace functional for pseudo-differential operators and the symplectic structure of Korteveg-Devries type equations, Invent. Math. 50 (1979), 219-248.

2. M.F. Atiyah, Convexity and commuting Hamiltonians, Bull. London Math. Soc. 16 (1982), 1-15.

3. A. M. Bloch, Estimation, principal components and Hamiltonian systems, Systems Control Lett., vol. 6, North-Holland, Amsterdam, 1985, pp. 103108.

4. _ Steepest descent, linear programming and Hamiltonian Flows, Contemporary Math. (to appear).

5. A. M. Bloch, H. Flaschka, and T. Ratiu, A convexity theorem for isospectral sets of Jacobi matrices in a compact Lie algebra, Duke Math. J. (to appear).

6. A. M. Bloch, R. W. Brockett, and T. Ratiu, Completely integrable gradient flows (to appear).

7. M. R. Bremner, R. V. Moody, and J. Patera, Tables of dominant weight multiplicities for representations of simple Lie algebras, Marcel Dekker, New York, 1985. 
8. R. W. Brockett, Least squares matching problems, Linear Algebra Appl. 122/123/124 (1989), 761-777.

9. __ Dynamical systems that sort lists and solve linear programming problems, Proc. 27th IEEE Conf. on Decision and Control, IEEE, New Jersey, 1988, pp. 799-803.

10. C. I. Byrnes and J. C. Willems, Least squares estimation, linear programming and momentum, unpublished manuscript, 1984.

11. M. T. Chu, The generalized Toda lattice, the $Q R$-algorithm and the centre manifold theory, SIAM J. Algebraic Discrete Methods 5 (1984), 187-201.

12. M. W. Davis, Some aspherical manifolds, Duke Math. J. 5 (1987), 105139.

13. P. Deift, T. Nanda, and C. Tomei, Differential equations for the symmetric eigenvalue problem, SIAM J. Numer. Anal. 20 (1983), 1-22.

14. H. Flaschka, The Toda lattice, Phys. Rev. B(3) 9 (1976), 1924-1925.

15. D. Fried, The cohomology of an isospectral flow, Proc. Amer. Math. Soc. 98 (1986), 363-368.

16. V. Guillemin and S. Sternberg, Convexity properties of the moment mapping, Invent. Math. 67 (1982), 491-513.

17. $\ldots$ On the method of Symes for integrating systems of the Toda type, Lett. Math. Phys. 7 (1983), 113-115.

18. A. Horn, Doubly stochastic matrices and the diagonal of a rotation matrix, Amer. J. Math. 76 (1956), 620-630.

19. B. Kostant, The principal three-dimensional subgroup and the Betti numbers of a complex simple Lie group, Amer. J. Math. 81 (1959), 973-1032.

20. On convexity, the Weyl group and the Iwasawa decomposition, Ann. Sci. École Norm. Sup. (4) 6 (1973), 413-455.

21. Adv. in Math. 34 (1979), 195-338.

22. _ Quantization and representation theory, in Representation theory of Lie groups, London Math. Soc. Lecture Note Ser., Cambridge Univ. Press, New York, vol. 34, 1979, pp. 91-150.

23. J. Lagarias, Monotonicity properties of the generalized Toda flow and $Q R$ flow, preprint, 1988.

24. I. G. MacDonald, Algebraic structure of Lie groups, in Representation theory of Lie groups, London Math. Soc. Lecture Note Ser. Cambridge University Press, New York, vol. 34, 1979, pp. 91-150.

25. J. Moser, Finitely many mass points on the line under the influence of an exponential potential, Batelles Recontres, Springer Notes in Physics, Springer-Verlag, Berlin, 1974, pp. 417-497.

26. W. W. Symes, Hamiltonian group actions and integrable systems, Phys. D 1 (1980), 339-376.

27. __ Systems of Toda type, inverse spectral problems and representation theory, Invent. Math. 59 (1982), 13-51.

28. - The $Q R$ algorithm and scattering for the nonperiodic Toda lattice, Phys. D 4 (1982), 275-280.

29. M. Toda, Studies of a non-linear lattice, Phys. Rep. 8 (1975), 1-125. 
30. C. Tomei, The topology of isospectral manifolds of diagonal matrices, Duke Math. J. 51 (1984), 981-996.

31. P. van Moerbeke, The spectrum of Jacobi matrices, Invent. Math. 37 (1976), 45-81.

Mathematical Sciences Institute, Cornell University, Ithaca, New York 14853 and Department of Mathematics, Ohio State University, Columbus, OHIO 43210

Division of Applied Sciences, Harvard University, Cambridge, MassaCHUSETTS 02138

Mathematical Sciences Research Institute, 1000 Centennial Drive, Berkeley, CAlifornia 94720 and Department of Mathematics, University of California, Santa Cruz, California 95069 
